Title: The Local response of El Niño events and changing disease distribution in Tanzania.

Main Author: Rachael Reynolds BSc.

Co-Authors: Dr Gina Cavan, Dr Mark Cresswell

Acknowledgements: Thanks to the British Atmospheric Data Centre, which is part of the NERC National Centre for Atmospheric Science (NCAS), for providing access to the Met Office MIDAS dataset, of which the appropriate scientific papers are referenced in this work.

\title{
Abstract
}

Climate is a key determinant affecting a number of disease pathogen lifecycles and disease transmission, particularly within tropical climates such as that experienced within Tanzania. Over recent decades, climatically related diseases such as malaria, Chikungunya and Bacterial meningitis have shown notable changes in their spatial distribution, where instances of both re-emerging in areas and expanding beyond previously known boundaries have been recorded. The unpredicted change in disease distribution already experienced within Tanzania has placed a significant burden upon health systems and available resources and whilst a number of factors are involved, climate remains the least understood aspect within these epidemiological alterations. Here we examine how climate extremes, particularly El Niño events, influence key environmental and climatic elements which promote epidemiological expansion.

This study investigates the baseline climatology in five of Tanzania's varying climatological regions using the Met Office MIDAS dataset, 1985 to 1995. This aims to characterise the average climate and investigate the impacts of El Niño on the climatology of these regions, and explore associated changes in disease distribution to allow any identified future changes in climate to be put into context. Years 1997 and 2015 are used to examine the climate extremes imposed by El Niño events through statistical comparison methods. The results demonstrate that average climate conditions vary beyond that previously documented, with each region of Tanzania responding differently to the onset of El Niño, thus potentially spurring a spatially variable disease response. These results are particularly for areas of greater climatic and environmental sensitivity within Tanzania. A relationship which when once further understood could be applied to more local analysis and aid in predicting future outbreaks within Tanzania. 


\section{The local response of El Niño events and changing disease distribution in Tanzania.}

\section{Introduction}

Climatic conditions play a key role in the formation of suitable habitats and conditions for disease transmission and epidemic outbreaks to occur (Pandya et al., 2015). Vector based diseases such as malaria, are particularly influenced by climatic conditions due to the temperature dependent nature of mosquitoes, the dominant transmitter of malaria, as well as the vectors habitat suitability (Killeen and Chitnis, 2014). Airborne diseases, such as meningococcal meningitis, are also highly influenced by climatic conditions, with strong epidemic relationships associated with low absolute humidity values and dry conditions (Cheesbrough et al., 1995). These relationships are mathematically characterised through slight abstract variations of the epidemiological triangle demonstrated in Figure 1 (Diekmann and Heesterbeek, 2000).

The resurgence and changing dynamics of malaria and meningococcal meningitis in relation to climate is of particular concern given the detrimental impacts of these diseases on both individual and national levels for countries like Tanzania (Bhatt et al., 2015). Meningococcal meningitis has comparatively less impact upon Tanzania in terms of disease burden at present, however, there is considerable concern about its resurgence due to recent epidemics and changing dominance of serotypes causing the disease (Molesworth et al., 2003). Changes in spatial distribution of disease have already been observed in Tanzania , including the documentation of unexpected epidemics being caused by different serogroups of the disease to which local populations have no natural immunity. These events are becoming more widely observed across the African meningitis belt, thus suggesting a potential change in local climatic conditions, requiring further examination (Halperin et al., 2012).

Malaria is caused by the virulent parasite plasmodium (P.Falciparum), accounting for over $30 \%$ of the national disease burden in Tanzania (Winskill et al., 2011). Malaria thrives in equatorial temperatures, where adequate provisions of water, vegetation and blood meals (humans) are present (Drakeley et al., 2005). Higher temperatures generally enhance the rate of development of both the parasite and the vector causing a mosquito population boom and thus pose a greater threat to local residents (Campbell-lendrum et al., 2015). However, current knowledge on the nature and drivers of changing endemicity of both malaria and meningitis in sub-saharan Africa remains relatively weak, supporting the case for further investigation into the drivers of these observed changes (Bhatt et al., 2015; Shayo et al., 2015).

Malaria is endemic to Tanzania with the exception of highland areas (above 1500m) which experiences infrequent epidemic outbreaks. Malaria has historically been mostly absent above this elevation due to higher altitudes experiencing lower temperatures than lower altitudes as a result of changing atmospheric characteristics with height (Chabot-Couture et al., 2014; Met Office, 2011). However, recent evidence suggests this is changing as a direct result of increasing temperatures at higher elevations, providing increasingly suitable conditions for transmission vectors (Jones et al., 2007). Mean temperatures in Tanzania have been observed to vary from $25^{\circ} \mathrm{C}$ in high altitude locations such as Mbeya (1704m) up to $32^{\circ} \mathrm{C}$ in lowland areas such as Dar es Salaam (55m) (TMA, 2014). Average temperatures at Mbeya are considered too low and varied to support vector growth and development, whereas average temperatures at Dar es Salaam are considered to be optimum at present (Mordecai et al., 2013; Parham and Michael, 2010).

Further to temperature, precipitation is a key factor in supporting vector growth and development through the provision of mosquito larvae habitats (Gwitira et al., 2015). Tanzania is characterised by two major rainfall regimes, where northern Tanzania experiences a bimodal regime with the south experiencing a unimodal rainfall regime. Dominant controlling factors in the onset and severity of these rainfall periods and thus malaria are the movement of the inter-tropical convergence zone (ITCZ) the Indian Ocean dipole and the EI Nino Southern oscillation (ENSO) (Basalirwa et al., 1999; Mapande and Reason, 2005). In addition, relative humidity of at least $60 \%$ is considered a requirement for malaria transmission as well as impacting upon bacterial meningitis, which also 
changes with elevation due to its relationship to temperature and air moisture (Duane et al., 2008). Overall, few studies have been conducted on malaria-climate relationships in Tanzania and other Eastern African countries, thus requiring further assessment.

ENSO events profoundly impact precipitation and temperature seasonality in the East African highlands, resulting in exceptionally heavy rainfall and raised temperatures (Lindsay et al., 2000; Nicholson and Kim, 1997). As a result, short term epidemiological changes have been observed and linked to the large-scale synoptic weather events of El Niño and La Niña, although more predominantly El Niño (Kovats et al., 2003). Whilst ENSO events have been linked to changing malaria distribution in Tanzania, conflicting results are evident. Lindsay et al., (2000) observed a decline in malaria infection following the 1997-98 ENSO event, in contrast to a comprehensive review by Kovats et al., (2003) which demonstrates multiple sources supporting increased malaria infection following the 1997-98 ENSO event. This has prompted the need to investigate the individual weather elements associated with ENSO rather than just using ENSO events alone (Jones et al., 2007; Kovats et al., 2003; Lindsay et al., 2000). Hence, examining changes in local climatic conditions experienced under EI Niño could provide an indication of areas sensitive to local climatic changes and thus by association, disease distribution in the future (Poveda et al., 2011).

This study aims to examine baseline local climatic conditions for selected stations within Tanzania, examining the extent of variance in response to two categorically strong El Niño events. It is anticipated this will provide an insight into areas which may be more likely to experience heightened local climatic variance in response to extreme climatic events such as ENSO, and thus potential local changes in disease dynamics. This has been achieved through the following objectives.

- Quantify the baseline climatic components of Tanzania, gaining an understanding of average annual conditions.

- Investigate the impact of extreme climate events through the onset of categorically strong EI Niño events.

- Assess the impact of El Niño events on the local climate conditions at five station locations across Tanzania.

- Consider how the observed changes could impact disease and disease environments.

It is important to note that whilst climate is a known driver of changing endemicity, socio-economic changes and population migration also play important roles, however only climatic variables are examined here. 


\section{Methodology}

\section{Datasets Used}

Five stations were selected to cover a range of environments using factors known to contribute to disease spread, as outlined in Table 1. Meteorological data for the stations was supplied by the British Atmospheric Data Centre (BADC) and collected through the Met Office Integrated Data Archive System (MIDAS). Locations of the five stations are shown in Figure 2.

Years 1985 through to 1995 were used to represent a baseline climatology for Tanzania. Whilst 30 years or more are preferred to represent a climatological average, this was not possible due to many stations only commencing data recording operations in 1983. Initial issues with irregular data entries in the early years of station operations warranted some years to be omitted. Furthermore, an El Niño year of interest for this study (1997) occurred shortly after data records began, thus limiting the baseline climate period to ten years.

El Niño years 1997 and 2015 were chosen to assess the impact of changing global conditions on the Tanzanian climate due to their categorically strong Southern Oscillation Index (SOI) values.

\section{Analytical Process}

Figure 3 demonstrates the workflow process used to assess each station dataset. Baseline climatology (1985-1995), 1997 and 2015 were each individually assessed using descriptive statistics before being combined and compared via ANOVA analysis. ANOVA tests were applied to determine whether there was any statistically significant difference between the climatological baseline (1985 $1995)$ and the two EI Niño years (1997 \& 2015). Where ANOVA tests concluded a statistically significant difference was present, then Post-hoc Tukey and Bonferroni tests were conducted to examine which month(s) demonstrated a statistically significant change. No further tests were carried out if ANOVA showed no significant difference. Tukey results were used as the dominant accuracy indicator despite requiring equal sample sizes, an aspect addressed in using synoptic hourly data only, it still performs to a reasonable degree of accuracy if sample sizes are not equal. Bonferroni tests were run to provide further evidence to support the statistical outcome (Ekstrøm and Sørensen, 2015).

Despite quality assurance checks performed on MIDAS datasets by the UK Met Office (UKMO) some outliers were identified in the form of abnormally high temperatures when the dataset was manually checked for any influential outliers (Met Office, 2016). This included temperature records of $99^{\circ} \mathrm{C}$ and dew points yielding relative humidity values above $100 \%$ which given the geographical location was deemed highly improbable.

In order to mass identify non-obvious outliers the standard deviation was calculated and multiplied by 4 , where anything outside of the $4 x$ standard deviation was removed (Figure 4). This method of identifying and removing outliers is common practice (Weisheimer and Palmer, 2014). This method was only applied to temperature and humidity datasets as a result of these parameters being statistically, normally distributed. Rainfall outliers were removed manually and discretion applied. For example, values depicting $601 \mathrm{~mm}$ of rainfall in one synoptic hour period (6 hours) seemed unreasonable for the area when assessed against local trends. The non-normal distribution of the data made this unsuitable to apply a $4 x$ standard deviation rule as potential natural variability could have been removed.

Relative humidity was initially calculated using temperature and dew point records provided within the station data and calculated using Equation 1 (Figure 3) and the constant values provided in Table 2. Whilst relative humidity is commonly reported, absolute humidity provides more comparable results when examining diseases such as bacterial meningitis. Relative humidity was converted using equations 2, 3 and 4 (Figure 3).

\section{Results \& Discussion}




\section{Baseline Climatology (1985-1995)}

Mean monthly temperatures vary across stations within this study (Figure 5, Table 3). Similar seasonal trends in temperature are seen across all stations, with the exception of Mwanza where the influence of complex mesoscale circulation patterns in the Lake Victoria basin alter local climatic conditions, thus impacting this particular station (Anyah and Semazzi, 2004). Mean monthly absolute humidity depicts a less exacerbated seasonal trend (Figure 8, Table 5), with Mwanza displaying a slightly different regime. As with temperature a clear altitudinal relationship can be seen with Mbeya (1704m above sea level) experiencing the overall lowest mean monthly absolute humidity which drops below $10 \mathrm{~g} / \mathrm{m}^{3}$ from June to September, a key value associated with the onset of bacterial meningitis (Cheesbrough et al., 1995; Pandya et al., 2015). Dar es Salaam (55m above sea level) experiences the highest absolute humidity.

Differences in rainfall regimes are clearly seen in the mean monthly rainfall total (Figure 6). Dodoma and Mbeya display a unimodal rainfall regime, and Dar es Salaam, Kilimanjaro and Mwanza all display a bimodal pattern, with start times varying in relation to the movement of the inter-tropical convergence zone (ITCZ) (Gaidet et al., 2012). Dar es Salaam experiences the greatest variation in rainfall (Table 4), which peaks in April at approximately $12 \mathrm{~mm}$ per day. Dar es Salaam's coastal location lends to the interpretation that the movement of clouds over the ocean deposit significant amounts upon reaching the Tanzanian landmass, which is further dictated by the movement of the ITCZ. Generally, there is a clear reduction of rainfall between June and September for all stations, with some experiencing minimal to zero rainfall for this period, notably Dodoma and Mbeya.

\section{Statistical Comparison between the Baseline Climatology and El Niño Conditions}

In order to investigate the impact of El Niño events on climatic variables, comparisons were made between seasonal differences in each climatic factor (Figures 8 - 10). Furthermore, Anova and Tukey analysis were carried out to examine which months and climatic variables experienced statistically significant differences between years (Table 6 -10). Dar es Salaam displays the greatest statistically significant change in climatic variables under EI Niño, meaning the event significantly impacted upon the local climate in Dar es Salaam, in particular temperature and humidity (Table 6). Whilst no discernible pattern can be identified due to the homogenous change observed with temperature and humidity a clear shift in rainfall seasonality is observed in both plotted trends and statistics, supporting a potential shift in seasonality of malaria transmission (Figure 10a, Table 6). Results suggest Dar es Salaam's climate is particularly sensitive to large scale synoptic processes and thus could potentially be at risk of experiencing significant climatic changes under future conditions in comparison to the other four locations. Further analysis into the differing reactions to El Niño conditions are recommended.

Humidity values demonstrated the greatest amount of statistical change out of the three climatic variables examined over three years. Whilst Dar es Salaam demonstrated the greatest change in absolute humidity, Mbeya also depicted significant humidity alterations for 2015. Mbeya's baseline absolute humidity demonstrates suitable conditions for meningococcal meningitis of $10 \mathrm{~g} / \mathrm{m}^{3}$ for 4 months per year with lowest observed values consistently being recorded below $10 \mathrm{~g} / \mathrm{m}^{3}$ (Figure 8 , Figure 11d). Whilst conditions seem appropriate for what is considered the most influential factor with regards to meningococcal meningitis distribution, no records for the disease have currently been observed in this location, suggesting other relevant climatic factors may not currently be suitable, yet further analysis should be conducted (Pandya et al., 2015). Furthermore, reductions in maximum absolute humidity and increased exacerbation in humidity trends from June - September further increase the suitability for meningococcal meningitis transmission within these months.

Dar es Salaam Airport

Dodoma

Kilimanjaro Airport

Mbeya 


\section{Conclusion}

This study has developed a baseline climatology useful for climate-related studies in Tanzania. Whilst limitations were encountered due to limited data availability and differing observation numbers, methods were developed to reduce the impact of these limitations on the study. EI Niño events have a statistically significant impact upon disease sensitive climatic factors, with some areas e.g. Dar es Salaam, demonstrating greater sensitivity of response to others such as Dodoma. Overall, no discernible pattern of change can be identified between EI Niño years or location. However, clear trends in baseline changes were observed through changing minima and maxima of temperature and absolute humidity, where overall minimum values appear to unanimously increase under El Niño. Dar es Salaam warrants closer investigation into the impacts of potential future climate change on disease given the evidence presented here. Evidence from this study and the literature also provides compelling reasoning for closer examination of Mbeya, an area which at present would be classified as being on the ecological boundary of mosquito survival and thus potentially malaria and meningococcal meningitis transmission (Pandya et al., 2015; Parham and Michael, 2010). 


\section{References}

Anyah RO and Semazzi FHM (2004) Simulation of the sensitivity of Lake Victoria basin climate to lake surface temperatures. Theoretical and Applied Climatology 79(1-2): 55-69.

Basalirwa CPK, Odiyo JO, Mngodo RJ, et al. (1999) The climatological regions of Tanzania based on the rainfall characteristics. International Journal of Climatology 19(1): 69-80.

Bhatt S, Weiss DJ, Cameron E, et al. (2015) The effect of malaria control on Plasmodium falciparum in Africa between 2000 and 2015. Nature: 1-9.

Campbell-lendrum D, Manga L, Bagayoko M, et al. (2015) Climate change and vector-borne diseases : what are the implications for public health research and policy? Philosophical Transactions of the Royal Society B: Biological Sciences 370.

Chabot-Couture G, Nigmatulina K and Eckhoff P (2014) An environmental data set for vector-borne disease modeling and epidemiology. PloS one 9(4): 1-17.

Cheesbrough J. S, Morse A. P and Green S. D (1995) Meningococcal meningitis and carriage in western Zaire: a hypoendemic zone related to climate? Epidemiology and infection 114(1): 7592.

Diekmann O (1996) Mathematical epidemiology of infectious diseases. In: Mathematical Epidemiology of Infectious Diseases. Model Building, Analysis and Interpretation., pp. 201-207.

Drakeley CJ, Carneiro I, Reyburn H, et al. (2005) Altitude-dependent and -independent variations in Plasmodium falciparum prevalence in northeastern Tanzania. The Journal of infectious diseases 191: 1589-1598.

Duane W., Pepin N., Losleben M., et al. (2008) General Characteristics of Temperature and Humidity Variability on Kilimanjaro, Tanzania. Arctic, Antarctic and Apline Research 40(2): 323-334.

Ekstrøm CT and Sørensen H (2015) Introduction to Statistical Data Analysis for the Life Sciences. 2nd Ed. London: CRC Press.

Gaidet N, Caron A, Cappelle J, et al. (2012) Understanding the ecological drivers of avian influenza virus infection in wildfowl: a continental-scale study across Africa. Proceedings of the Royal Society B: Biological Sciences 279(1731): 1131-1141.

Gwitira I, Murwira A, Zengeya FM, et al. (2015) Modelled habitat suitability of a malaria causing vector (Anopheles arabiensis) relates well with human malaria incidences in Zimbabwe. Applied Geography 60: 130-138.

Halperin SA, Bettinger JA, Greenwood B, et al. (2012) The changing and dynamic epidemiology of meningococcal disease. Vaccine 30(2).

Jones AE, Wort U, Morse AP, et al. (2007) Climate prediction of EI Niño malaria epidemics in northwest Tanzania. Malaria Journal 6(1): 162.

Killeen GF. b GF and Chitnis N. d e N (2014) Potential causes and consequences of behavioural resilience and resistance in malaria vector populations: A mathematical modelling analysis. Malaria Journal 13(1): 1-16.

Kovats RS, Bouma MJ, Hajat S, et al. (2003) El Niño and health. The Lancet 362(9394): 1481-1489.

Lindsay SW, Bødker R, Malima R, et al. (2000) Effect of 1997-98 El Nino on highland malaria in Tanzania. Lancet 355: 989.

Mapande AT and Reason CJC (2005) Interannual rainfall variability over western Tanzania. International Journal of Climatology 25(10): 1355-1368.

Met Office (2011) National Meteorological Library and Archive Fact sheet No . 13 - Upper air observations and the tephigram.

Met Office (2016) Met Office Surface Data Users Guide. 
Molesworth AM, Cuevas LE, Connor SJ, et al. (2003) Environmental risk and meningitis epidemics in Africa. Emerging Infectious Diseases 9(10): 1287-1293.

Mordecai EA, Paaijmans KP, Johnson LR, et al. (2013) Optimal temperature for malaria transmission is dramatically lower than previously predicted. Ecology Letters 16(1): 22-30.

NBS (2013) Tanzania in Figures, 2012. Dar es Salaam.

Nicholson SE and Kim J (1997) the Relationship of the EI Ni O-Southern Oscillation To African Rainfall. International Journal of Climatology 17: 117-135.

Pandya R, Hodgson A, Hayden MH, et al. (2015) Using weather forecasts to help manage meningitis in the West African Sahel. Bulletin of the American Meteorological Society 96(1): 103-115.

Parham PE and Michael E (2010) Modeling the effects of weather and climate change on malaria transmission. Environmental health perspectives 118(5): 620-626.

Poveda G, Estrada-Restrepo Ó a., Morales JE, et al. (2011) Integrating knowledge and management regarding the climate-malaria linkages in Colombia. Current Opinion in Environmental Sustainability 3(6): 449-460.

Shayo EH, Rumisha SF, Mlozi MRS, et al. (2015) Social determinants of malaria and health care seeking patterns among rice farming and pastoral communities in Kilosa District in central Tanzania. Acta Tropica 144: 41-49.

TMA (2014) Climate Change Projection for Tanzania. Dar es Salaam.

Vaisala (2013) Calculation formulas for humidity. Humidity Conversion Formulas, Helsinki.

Weisheimer A and Palmer TN (2014) On the reliability of seasonal climate forecasts. Journal of the Royal Society, Interface / the Royal Society 11(96): 20131162.

Winskill P, Rowland M, Mtove G, et al. (2011) Malaria risk factors in north-east Tanzania. Malaria journal 10(1): 98. 